\title{
Conditionally positive effect of the TetR-family transcriptional regulator AtrA on streptomycin production by Streptomyces griseus
}

\author{
Correspondence \\ Sueharu Horinouchi \\ asuhori@mail.ecc.u-tokyo.ac.jp
}

Received 25 October 2007

Revised 2 December 2007

Accepted 17 December 2007
Setsu Hirano, Katsuyuki Tanaka, Yasuo Ohnishi and Sueharu Horinouchi

Department of Biotechnology, Graduate School of Agriculture and Life Sciences, The University of Tokyo, Bunkyo-ku, Tokyo 113-8657, Japan

\section{INTRODUCTION}

The genus Streptomyces consists of Gram-positive, filamentous, soil-dwelling bacteria that are characterized by complex morphological differentiation resembling that of fungi and by the ability to produce a wide variety of secondary metabolites. About two-thirds of all known biologically active substances, including antibiotics, are produced by streptomycetes. The biosynthetic and regulatory genes for a certain secondary metabolite are generally found in a gene cluster that includes a regulatory gene encoding a pathwayspecific transcriptional activator. The pathway-specific transcriptional activator acts as a master switch for biosynthesis of the respective secondary metabolite. Therefore, a variety of external signals, such as nutrient conditions and physiological conditions, are probably gathered to the promoter of a pathway-specific transcriptional activator gene, for example, actII-ORF4 for the actinorhodin biosynthesis gene cluster in Streptomyces coelicolor A3(2) (Fernández-Moreno et al., 1991) and strR

The GenBank/EMBL/DDBJ accession number for the nucleotide sequence of atrA-g is AB365454. for the streptomycin biosynthesis gene cluster in Streptomyces griseus (Retzlaff \& Distler, 1995; Tomono et al., 2005).

We have long studied the A-factor (2-isocapryloyl-3Rhydroxymethyl- $\gamma$-butyrolactone) regulatory cascade that leads to secondary metabolism and morphological differentiation in S. griseus (Horinouchi, 2007; Horinouchi \& Beppu, 2007). As one of the target phenotypes controlled by A-factor, the regulation of streptomycin biosynthesis has been studied with a focus on the pathway-specific activator gene $s t r R$. The signal relay from A-factor to $s t r R$ is as follows. A-factor is gradually accumulated in a growth-dependent manner by the activity of AfsA, which is the key enzyme for A-factor biosynthesis (Kato et al., 2007). When the concentration of A-factor reaches a critical level at or near the middle of exponential growth (Ando et al., 1997), it binds the A-factor receptor protein (ArpA), which has bound and repressed the promoter of $a d p A$, and dissociates ArpA from the promoter, thus inducing transcription of adpA (Ohnishi et al., 1999). AdpA then activates a number of genes required for morphological differentiation and secondary metabolism, forming an AdpA regulon (Horinouchi, 2002; Ohnishi et al., 2005). strR was the first member of the AdpA regulon to be identified, as described below. 
We previously identified the strR promoter as an A-factordependent promoter (Vujaklija et al., 1991) and therefore conducted experiments to detect a predicted activator protein(s) that might bind the region upstream from the strR promoter. In this experiment, we detected four proteins that did bind the region in vitro (Vujaklija et al., 1993). One of them, which bound the region at approximately nucleotide position -270 relative to the transcriptional start point of $\operatorname{str} R$ as +1 , was produced in an A-factor-dependent manner and named AdpA (Afactor-dependent protein). We purified AdpA, cloned the gene, and found that adpA was a target gene of ArpA (Ohnishi et al., 1999). Because disruption of adpA resulted in no transcriptional activation of $s t r R$ and no production of streptomycin, AdpA was proved to be essential for $s t r R$ transcription (Ohnishi et al., 1999). Furthermore, we identified an additional AdpA-binding site at nucleotide position -50 in front of the $s t r R$ promoter and found that both AdpA-binding sites (positions -270 and -50 ) are necessary for full transcriptional activation of $s t r R$ by AdpA (Tomono et al., 2005). However, the three proteins other than AdpA that bound the region upstream from $s t r R$, and were apparently produced independently of A-factor, have not been studied any further. Therefore, whether or not these three proteins are involved in the regulation of the transcription of $s t r R$ has not been elucidated.

Recently, McDowall and his colleagues identified a transcriptional activator for actII-ORF4 encoding the pathway-specific transcriptional activator for the actinorhodin biosynthesis gene cluster in S. coelicolor A3(2) (Uguru et al., 2005). They named this TetR-family regulator AtrA (actinorhodin-associated transcriptional regulator). Here we call it AtrA-c to discriminate it from its homologue (AtrA-g) in S. griseus. AtrA-c binds two sites flanking the promoter of actII-ORF4. Disruption of $\operatorname{atr} A-c$, which is not associated with any secondary metabolite gene cluster, reduced production of actinorhodin but showed no detectable effects on production of other secondary metabolites, such as undecylprodigiosin and a calciumdependent antibiotic (CDA). Furthermore, they investigated whether AtrA-c orthologues have a role in regulating transcription of some pathway-specific transcriptional activators for secondary metabolites in disparate Streptomyces strains and showed that AtrA-c bound in vitro the region upstream from the strR promoter of $S$. griseus. In their following paper (Hong et al., 2007), they reported that expression of atrA-c in S. griseus caused DNA-binding-dependent reduction in streptomycin production and in the strR mRNA level. Because expression of atrA-c in S. griseus did not affect the adpA mRNA level, they proposed that the A-factor-ArpA-AdpA-StrR regulatory cascade represents only part of the full complexity of regulation of streptomycin biosynthesis in $S$. griseus. However, they did not characterize the function of the endogenous atrA-g gene and therefore it was unclear whether AtrA-g actually contributes to streptomycin biosynthesis in S. griseus.
The work by Uguru et al. (2005) and Hong et al. (2007) reminded us of the four proteins, including AdpA, that bound the region upstream from the $s t r R$ promoter. On the assumption that AtrA-g could be one of the three proteins other than AdpA, we started characterizing atrA-g to determine whether it plays a role in streptomycin production in S. griseus. Here we report that AtrA-g is non-essential for streptomycin production but has a conditionally positive effect.

\section{METHODS}

General recombinant DNA studies. All the bacterial strains, plasmids and media used were described previously (Kato et al., 2005; Hirano et al., 2006). Bennett agar without glucose [yeast extract, $0.1 \%$; meat extract (Kyokuto), $0.1 \%$; NZ amine, $0.2 \%$; agar, $2 \%$; $\mathrm{pH}$ 7.2] and SMM agar [glucose, 0.9\%; L-asparagine, 0.9\%; $\left(\mathrm{NH}_{4}\right)_{2} \mathrm{SO}_{4}, 0.2 \%$; Trizma base, $0.24 \%$; $\mathrm{NaCl}, 0.1 \% ; \mathrm{K}_{2} \mathrm{SO}_{4}$, $0.05 \% ; \mathrm{MgSO}_{4} .7 \mathrm{H}_{2} \mathrm{O}, 0.02 \% ; \mathrm{CaCl}_{2}, 0.01 \%$; trace element solution (Hopwood et al., 1985), $1 \%$; agar, $2 \%$; pH 7.2] containing $2.5 \mathrm{mM}$ $\mathrm{KH}_{2} \mathrm{PO}_{4}$ were used for streptomycin assay. The strategies used for gene disruption, alteration of the AtrA-g-binding site by PCR, gel mobility shift assay, DNase I footprinting and S1 nuclease mapping were also described previously (Yamazaki et al., 2000; Hirano et al., 2006).

S1 nuclease mapping. Total RNA was isolated with an RNAqueous kit (Ambion) from cells grown on cellophane on the surface of Bennett agar containing 1\% maltose. Hybridization probes were prepared by PCR with a pair of ${ }^{32} \mathrm{P}$-labelled and non-labelled primers. The PCR primers used for low-resolution S1 nuclease mapping were atrA-g-SLF and atrA-g-SLR ${ }^{*}$ for $a t r A-g$ and strR-S1F and strR-S1R ${ }^{*}$ for $\operatorname{strR}$ (Table 1). Primers indicated with an asterisk were labelled at the $5^{\prime}$ end with $\left[\gamma_{-}{ }^{32} \mathrm{P}\right]$ ATP by using T4 polynucleotide kinase before PCR. Primers atrA-g-SHF and atrA-g-SHR ${ }^{*}$ were used for highresolution $\mathrm{S} 1$ nuclease mapping of $\operatorname{atr} A-g$.

Production and purification of AtrA-g. The sequence (Met-1 to Ala-267) of AtrA-g was expressed as a fusion to a His-tag at the $\mathrm{N}$ terminus, as follows. The atrA-g sequence encoding Met-1 to Ala-267 was amplified by PCR with primers atrA-g-pF (containing SphI and NdeI sites) and atrA-g-pR (containing a BamHI site). The atrA-gcoding sequence was excised as an SphI-BamHI fragment and placed between the SphI and BamHI sites of pUC19. The absence of PCR errors was checked by nucleotide sequencing. The NdeIBamHI fragment was excised from this plasmid and placed between the NdeI and BamHI sites of pET16b, resulting in pET16batrA-g. Escherichia coli BL21(DE3) containing pLysS harbouring pET16b-atrA-g was cultured at $26.5{ }^{\circ} \mathrm{C}$ for $3 \mathrm{~h}$ in the presence of $1 \mathrm{mM}$ IPTG. AtrA-g with a His-tag at its $\mathrm{N}$ terminus was purified from the soluble fraction with a nickel-nitrilotriacetic acid spin column (Qiagen) according to the manual of the manufacturer. The AtrA-g protein was eluted by sodium phosphate buffer $[50 \mathrm{mM}$ $\mathrm{NaH}_{2} \mathrm{PO}_{4}, 300 \mathrm{mM} \mathrm{NaCl}, 250 \mathrm{mM}$ imidazole and $10 \%$ glycerol (pH 8.0)].

Gel mobility shift assay. The DNA fragments used for ${ }^{32} \mathrm{P}$-labelled probes were amplified by PCR and ${ }^{32} \mathrm{P}$-labelled with $\mathrm{T} 4$ polynucleotide kinase. Various regions upstream and in the coding sequence of atrA-g were used as ${ }^{32} \mathrm{P}$-labelled probes. Table 1 lists the primer sequences used for preparing these probes. Four probes, S1 to S4, were prepared as follows: strR-F1 and strR-R1 were used for probe S1; strR-F2 and strR-R2 for probe S2; strR-F3 and strR-R3 for probe S3; and strR-F4 and strR-R4 for probe S4. 
Table 1. Primers used in this study

\begin{tabular}{|c|c|c|}
\hline Gene and primer & Position & Sequence $\left(5^{\prime}-3^{\prime}\right)$ \\
\hline \multicolumn{3}{|l|}{$\operatorname{atrA}-g$} \\
\hline atrA-g-SLF & -99 to -80 & ACGATGTCCCGGTGCGGATC \\
\hline atrA-g-SHF & -204 to -185 & CGGCAAATTGTGTGACCGGA \\
\hline atrA-g-SHR & +106 to +87 & TGAATATGCATAACTTCCCC \\
\hline datrA-g-LF & +996 to +1026 & CCCAAGCTTCACGTGTTGCTGGACATCCTGCTGGAGGGGCTGCGGT \\
\hline datrA-g-RF & -1944 to -1915 & GCTCTAGAGACCGACCGTACTGACAGTCGCCGCCCGGT \\
\hline datrA-g-RR & +302 to +273 & CCCAAGCTTTCCTGTCGTCGCCGTTCCCGACGTCGTCGC \\
\hline atrA-g-pF & +237 to +266 & ACATGCATGCCATATGGGTTCGGCCGTTGCCGGACACGCCCCG \\
\hline atrA-g-pR & +1037 to +1009 & CGGGATCCGCCGGGCGCGACCGCAGCCCCTCCAGCA \\
\hline catrA-g-F & -374 to -345 & CGGAATTCCAGTCTCTCCTTCAACTTCCGTTGGAGACG \\
\hline catrA-g-R & +1476 to +1447 & CGGGATCCTCTTCCTGCTGCTGCTCGTTACCGCTCATC \\
\hline strR-R2 & +19 to -5 & AGAGCAATGCTTTCGCACTTCGCG \\
\hline strR-F3 & -225 to -202 & ATGTATTCGGCAATCAAACTGCGG \\
\hline strR-R3 & -50 to -71 & CCGCATGACCGAAAAGCGACCT \\
\hline strR-F4 & -68 to -46 & TCGCTTTTCGGTCATGCGGACAG \\
\hline strR-R4 & +136 to +117 & TTCACTTTCCTCGACCGCAC \\
\hline strR-S1F & -184 to -162 & AATGAAATCGCTGACAGGCGGTG \\
\hline strR-S1R & +218 to +199 & CGACATCCTCGCCGGCACTG \\
\hline strR-LmtF & -488 to -468 & CGGGATCCATCCCGGCGGCACGTATGGCC \\
\hline strR-LmtR & -138 to -159 & CCCAAGCTTAGCCAGCGCCTGCGCCCGATCA \\
\hline strR-RmtF & -131 to -101 & CCCAAGCTTGGCAGCGCTGGTCCTCTCCGCCCTGGAGGAT \\
\hline strR-RmtR & +334 to +314 & TCCGCGCATTTTGGTGGCGCG \\
\hline
\end{tabular}

DNase I footprinting. The method of DNase I footprinting was described previously (Yamazaki et al., 2000). For analysis of AtrA-gbinding sites, a ${ }^{32} \mathrm{P}$-labelled fragment was prepared by PCR with primers strR-F3 and strR-R3.

Alteration of the AtrA-g-binding sequence by PCR. Two mutations were introduced by PCR into the AtrA-g-binding site upstream of $s t r R$. The region upstream from the AtrA-g-binding site was amplified with primers strR-LmtF (containing a BamHI sequence at the $5^{\prime}$ end) and strR-LmtR (containing a HindIII sequence at the $5^{\prime}$ end), and digested with BamHI plus HindIII. The region downstream from the AtrA-g-binding site was amplified with primers strR-RmtF (containing HindIII and Aor51HI sequences at the $5^{\prime}$ end) and strRRmtR, and digested with HindIII plus SphI (position +305). The BamHI-HindIII fragment and the HindIII-SphI fragment were inserted between the BamHI and SphI sites of pUC19 by threefragment-ligation, generating pUC-mt-strR. The absence of PCR errors was checked by nucleotide sequencing. The GGAGGG and CGTTCC sequences in the AtrA-g-binding site were changed to HindIII (AAGCTT) and Aor51HI (AGCGCT) cleavage sequences, respectively. Mutated probe $\mathrm{mS} 2$ used for gel mobility shift assay was amplified by PCR using pUC-mt-strR as the template.

Gene disruption. For disruption of $a t r A-g$ on the chromosome, the atrA-g sequence was replaced by the neomycin resistance gene aphII. Briefly, a $2.2 \mathrm{~kb}$ sequence upstream of the atrA-g coding sequence and a $2.2 \mathrm{~kb}$ sequence downstream of the atrA- $g$ coding sequence, together with aphII, were assembled in pUC19. This mutagenic plasmid was linearized by digestion with DraI (Oh \& Chater, 1997) and introduced by transformation into $S$. griseus IFO13350, and neomycin $(10 \mu \mathrm{g}$ $\mathrm{ml}^{-1}$ )-resistant colonies were isolated. Correct disruption was checked by Southern hybridization with the atrA-g sequence (positions -1944 to +302 , prepared with primers datrA-g-RF and datrA-g-RR) and the aphII sequence as ${ }^{32} \mathrm{P}$-labelled probes against the chromosomal DNA digested with HindIII plus PvuII.

Construction of a complementation plasmid. For construction of pKU209-atrA-g, the atrA-g sequence with its promoter region was amplified by PCR with primers catrA-g-F (containing an EcoRI sequence at the $5^{\prime}$ end) and catrA-g-R (containing a BamHI sequence at the $5^{\prime}$ end), and digested with EcoRI plus BamHI. The EcoRI-BamHI fragment was inserted between the EcoRI and BamHI sites of pUC19, generating pUC-atrA-g. The absence of PCR errors was checked by nucleotide sequencing. The $P v u I I$ fragment was excised from pUCatrA-g and inserted in the SmaI site of pUC19. The PstI fragment including the atrA-g sequences was excised from this plasmid and inserted in the PstI site of pKU209, generating pKU209-atrA-g.

Streptomycin assay. The amount of streptomycin produced was measured by a bioassay using Bacillus subtilis ATCC 6633 as an indicator (Horinouchi et al., 1984).

\section{RESULTS}

\section{atrA orthologue (atrA-g) in S. griseus}

We searched the genome sequence of S. griseus IFO13350 (our unpublished data) for ORFs encoding an AtrA-c-like 
(a)

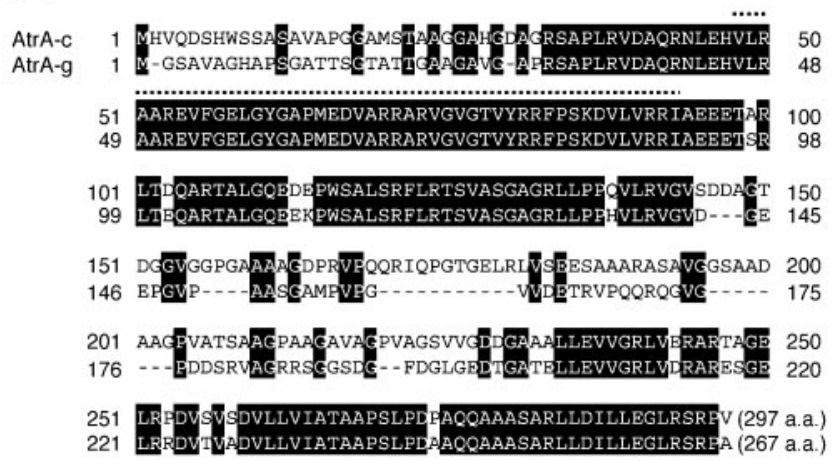

(b)

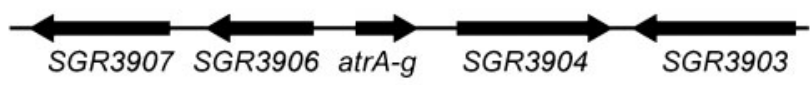

(c)

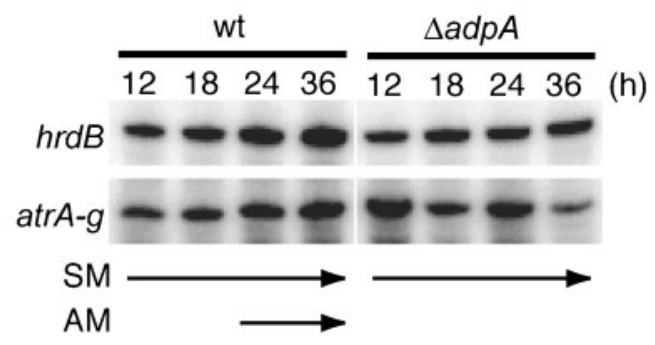

(d)
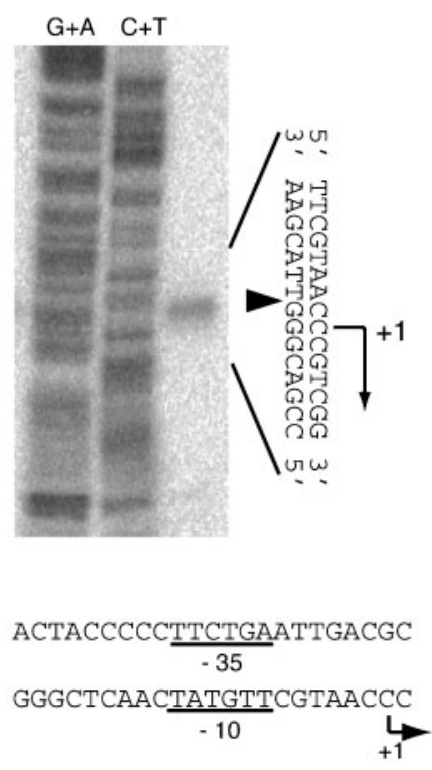

protein. A single ORF, designated atrA-g, which encoded a protein exhibiting end-to-end similarity to AtrA-c, was found. Fig. 1(a) shows the alignment of the amino acid
Fig. 1. Amino acid sequence of AtrA (a), gene organization in the neighbourhood of atrA-g (b), and transcriptional analysis of atrA-g (c, d). (a) Alignment of amino acid sequences of AtrA-c from $S$. coelicolor A3(2) and AtrA-g from S. griseus. The TetR-type DNAbinding domain is indicated with a dotted line. (b) Positions and directions of ORFs predicted by the $S$. griseus genome sequence are indicated by arrows. The predicted ORFs are: SGR3903, hypothetical protein; SGR3904, hypothetical protein; SGR3906, putative NADH dehydrogenase; and SGR3907, hypothetical protein. (c) Time-course of atrA-g transcription, as determined by low-resolution S1 mapping with RNA prepared from cells grown at $28{ }^{\circ} \mathrm{C}$ on cellophane on the surface of Bennett agar containing $1 \%$ maltose for the times indicated. As an internal control, transcription of $h r d B$ (constitutive) was also determined. SM, substrate mycelium; AM, aerial mycelium; wt, wild-type. Mutant $\triangle a d p A$ grew as substrate mycelium throughout growth. (d) Highresolution $\mathrm{S} 1$ mapping to determine the transcriptional start point of atrA-g. RNA prepared from mutant $\triangle a d p A$ grown at $28^{\circ} \mathrm{C}$ for $12 \mathrm{~h}$ on Bennett agar was used. The arrowhead indicates the position of the S1-protected fragment. The $5^{\prime}$ terminus of the mRNA was assigned to position +1 , because the fragments generated by the chemical sequencing reactions migrated $1.5 \mathrm{nt}$ further than did the corresponding fragments generated by $\mathrm{S} 1$ digestion of the DNA-RNA hybrids ( 0.5 residue from the presence of the $3^{\prime}$-terminal phosphate group and 1 residue from elimination of the $3^{\prime}$-terminal nucleotide). The nucleotide sequence, including the -35 and -10 elements of the atr $A-g$ promoter, is also shown. The transcriptional start point is indicated as +1 .

sequences of AtrA-c and AtrA-g. In these two proteins, the sequences of about 110 aa in the $\mathrm{N}$-terminal portion are highly homologous to each other. This region contains a TetR-type DNA-binding domain (46 aa), shown by a dotted line in Fig. 1(a). The amino acid sequences of the DNA-binding domains are identical between AtrA-g and AtrA-c, suggesting that both proteins would recognize and bind the same DNA sequence. The C-terminal regions of about 70 aa are also highly homologous to each other, although the function of this domain is unknown. The gene organization in the neighbourhood of atrA-g is shown in Fig. 1(b). atrA-g is located between the ORFs encoding a putative NADH dehydrogenase (SGR3906) and a hypothetical protein (SGR3904). This gene organization is the same in the region around atrA-c in S. coelicolor A3(2) (Bentley et al., 2002), indicating that atrA-g is the S. griseus orthologue of atrA-c in S. coelicolor A3(2).

We analysed the transcription of atrA-g by $\mathrm{S} 1$ nuclease mapping by using RNA prepared from cells grown on cellophane on the surface of Bennett agar containing $1 \%$ maltose (Fig. 1c). Under these conditions, S. griseus grew as substrate mycelium at 12 and $18 \mathrm{~h}$, and as a mixture of substrate mycelium and aerial mycelium at 24 and $36 \mathrm{~h}$. The $h r d B$ gene, which is transcribed throughout growth, was used as an internal control to check the integrity and amount of mRNA used. We found that atrA-g was constitutively transcribed throughout growth not only in the wild-type strain but also in an adpA-disrupted strain (mutant $\triangle a d p A$ ). The transcriptional start point was 
determined to be $236 \mathrm{nt}$ upstream of the translational start codon, as determined by high-resolution S1 nuclease mapping (Fig. 1d). We propose that the -35 and -10 sequences are $5^{\prime}$-TTCTGA-3' and 5' ${ }^{\prime}$-TATGTT-3', respectively, with a space of $17 \mathrm{nt}$ between them (Fig. 1d). The -35 and -10 sequences are similar to those ( $5^{\prime}$-TTGACR- $3^{\prime}$ and 5'-TAGRRT-3', respectively; $\mathrm{R}=\mathrm{A}$ or $\mathrm{G})$ recognized probably by the house-keeping sigma factor $\mathrm{HrdB}$ (Strohl, 1992). This fact is consistent with the constitutive transcription of atrA-g.

\section{Binding of AtrA-g to the region upstream from the strR promoter}

For purification of AtrA-g as a His-tagged protein from $E$. coli, we constructed pET16b-atrA-g carrying the atrA-g sequence under the control of the T7 promoter. The recombinant AtrA-g protein (His-AtrA-g), having the structure Met-Gly-His ${ }_{10}$-Ser ${ }_{2}$-Gly-His-Ile-Glu-Gly-ArgHis-AtrA-g, was produced in the soluble fraction of E. coli harbouring pET16b-atrA-g and purified with a nickelnitrilotriacetic acid spin column (Fig. 2a). Using this protein, we examined whether AtrA-g bound the region upstream from the $s t r R$ promoter by this assay. We designed four ${ }^{32} \mathrm{P}$-labelled probes (S1 to $\mathrm{S} 4$ ) for various regions and used them for gel mobility shift assay (Fig. 2b). Two of the four probes tested, S2 (nucleotide positions -344 to +19 , relative to the transcriptional start point of strR as +1 ) and S3 (positions -225 to -50 ), gave a single retarded signal (Fig. 2b). A very faint signal observed when a large amount of His-AtrA-g was used for probe S1 presumably resulted from non-specific binding. Because only one retarded signal was detected with the two probes, a single AtrA-g-binding site in the overlapping region was expected.

To determine the exact location of the AtrA-g-binding site, we performed DNase I footprinting with a ${ }^{32} \mathrm{P}$-labelled probe covering the region from -225 to -50 . AtrA-g protected from DNase I digestion a sequence from positions -117 to -142 on the sense and antisense strands (Fig. 2d). In this region, there was an imperfect inverted repeat sequence, as shown in Fig. 2(d). This AtrA-gbinding site was the same as the AtrA-c-binding site determined by Uguru et al. (2005), consistent with the fact that the TetR-type DNA-binding domains of AtrA-g and AtrA-c are completely conserved. It should be noted that the binding site of AtrA-g overlaps with that (positions -188 to -74 ) of one of the three proteins which we previously detected by gel mobility shift assay as being able to bind the region upstream of strR (Vujaklija et al., 1993). AtrA-g presumably corresponds to this protein.

\section{An inverted repeat sequence as an AtrA-g-binding site}

To confirm the importance of the imperfect inverted repeat as the AtrA-g-binding site, we introduced mutations into the sequence by site-directed mutagenesis, as shown in Fig. 2(c). One half of the palindrome (positions -137 to -132) was changed to a HindIII recognition sequence and the other half of the palindrome was changed to an Aor $51 \mathrm{HI}$ recognition sequence. The effect of the mutation on AtrA-g binding was examined by gel mobility shift assay (Fig. 2c). The ${ }^{32} \mathrm{P}$-labelled probe (mS2; positions -344 to +19 ) containing the mutation gave no retarded signal, whereas a similar probe (S2) containing the intact AtrA-gbinding sequence gave a distinct signal (Fig. 2b). These data showed that the imperfect inverted repeat sequence from positions -123 to -137 was the AtrA-g binding site.

\section{Simultaneous binding of AdpA and AtrA-g to the region upstream from the strR promoter}

AdpA binds two sites upstream from the $s t r R$ promoter, at approximately positions -270 and -50 , and activates the transcription of strR (Tomono et al., 2005). The AtrA-gbinding site was found between the two AdpA-binding sites (Fig. 3a). To determine whether AdpA and AtrA-g can simultaneously bind the DNA fragment containing the respective binding sites, we performed a gel mobility shift assay using the ${ }^{32} \mathrm{P}$-labelled probe covering the region from -344 to +19 (probe S2). The binding of two molecules of AdpA dimer to this fragment was confirmed (Fig. 3b, lanes 1-5). Although the AdpA-DNA complex seemed to be readily aggregated and stacked in the gel well, two retarded signals were detected, as indicated by arrows. We next examined the binding of AdpA to this probe S2 in the presence of an excess of AtrA-g (Fig. 3b, lanes 6-10). In the absence of AdpA, almost all the probe DNA fragments were retarded by AtrA-g to give a single distinct signal (Fig. 3b, lane 6). When AdpA at different concentrations was added to the reaction mixture (Fig. 3b, lanes 7-10), two additional retarded signals were detected. Because an excess of AtrA-g sufficient to bind all the probe DNA fragments was present in these reaction mixtures and because the AtrA-g-binding site was far enough apart from the two AdpA-binding sites to avoid competitive binding, almost all the probes were assumed to be bound by at least AtrA-g under the experimental conditions. We therefore assumed that the fast-moving one of the two additional retarded signals represented a ternary complex consisting of DNA, AtrA-g and one molecule of AdpA dimer. The upper one presumably represented a ternary complex consisting of DNA, AtrA-g and two molecules of AdpA dimer. This result showed that two molecules of AdpA dimer and one molecule of AtrA-g were capable of simultaneously binding the DNA fragment.

In this experiment, we noticed that the presence of AtrA-g in the reaction mixture lowered the minimum concentration of AdpA to bind the DNA fragment. For example, when $0.1 \mu \mathrm{g}$ AdpA was used, no retarded signal was detected in the absence of AtrA-g (Fig. 3b, lane 2), whereas half of the DNA fragment bound by AtrA-g was further retarded by the binding of AdpA (Fig. 3b, lane 7). These 
(a)

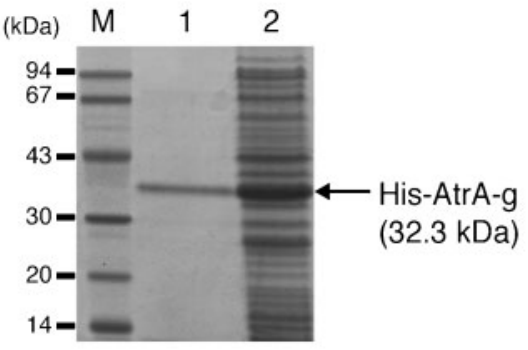

(b)

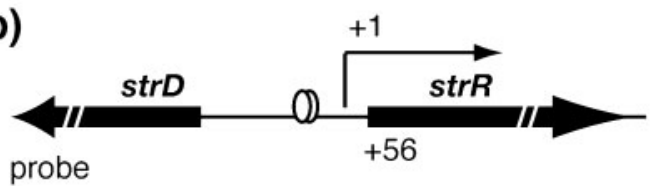

probe

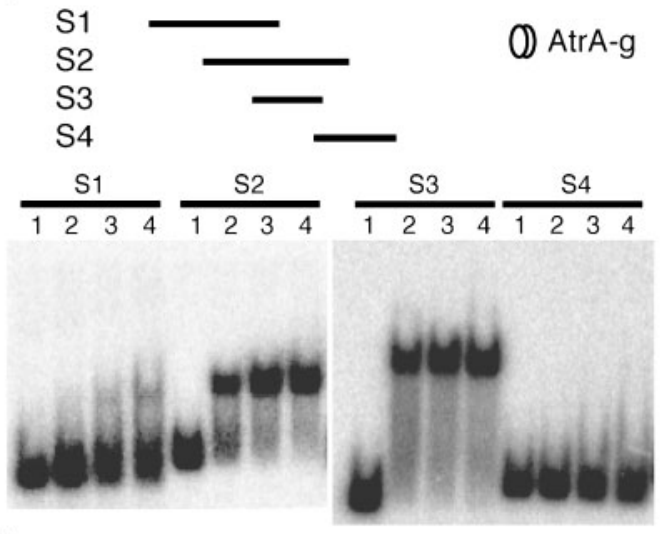

(c)

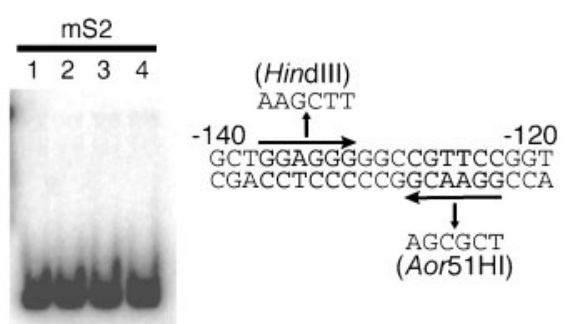

(d)
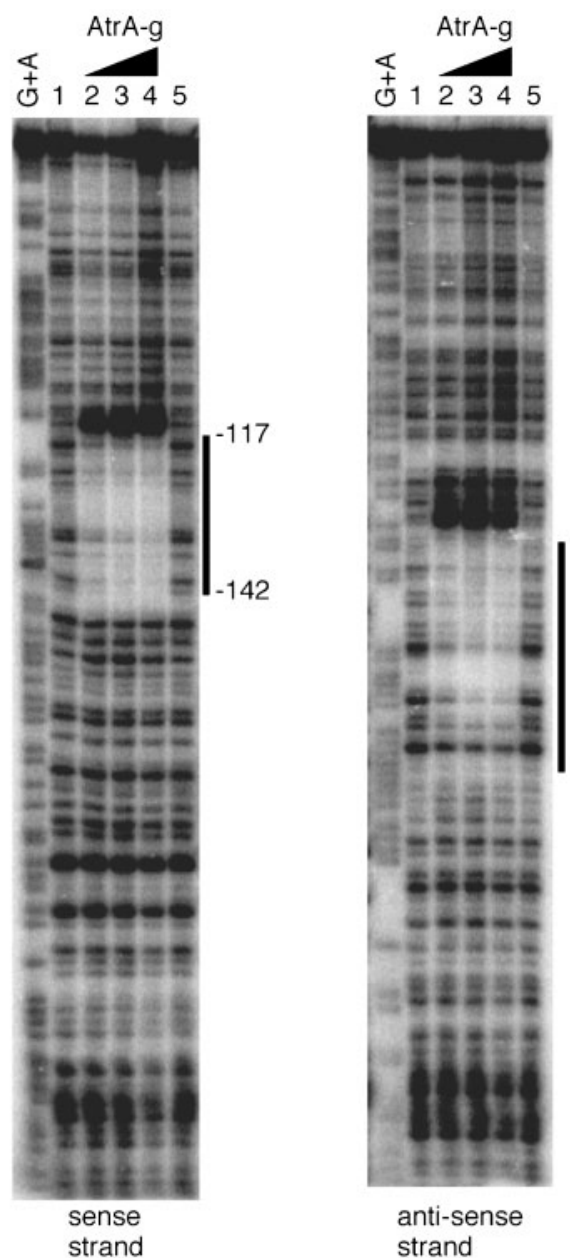

17 42

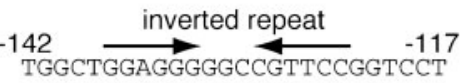

Fig. 2. Purification of His-tagged AtrA-g from E. coli (a), gel mobility shift assays to determine the binding site of AtrA-g (b), generation of mutations in the inverted repeat in the region upstream of the strR promoter (c), and DNase I footprinting to determine the AtrA-g binding site (d). (a) E. coli harbouring pET16b-atrA-g was grown in the presence of IPTG, and the Histagged AtrA-g protein was purified with a nickel-nitrilotriacetic acid spin column. The soluble fraction (lane 2) and the purified sample (lane 1), together with molecular size markers (lane M), were electrophoresed on an SDS-polyacrylamide gel. (b) Gel mobility shift assays with purified AtrA-g and four ${ }^{32} \mathrm{P}$-labelled probes, S1 to S4. The amounts of AtrA-g used were $0.2 \mu \mathrm{g}$ (lane 2), $0.4 \mu \mathrm{g}$ (lane 3) and $0.8 \mu \mathrm{g}$ (lane 4). Lane 1 was a control lane in which no AtrA-g was present. AtrA-g is illustrated as a dimer because it probably binds the inverted repeat in this form. (c) Gel mobility shift assay was performed with mutated probe, mS2. Mutations were introduced into the AtrA-g-binding sequence by PCR to replace the six nucleotides with Hindlll and Aor51HI recognition sequences, as shown. (d) DNase I footprinting assays were performed on the sense and antisense strands. The amounts of $\mathrm{H}$-AtrA-g used in lanes $1,2,3,4$ and 5 were $0,0.2,0.4,0.8$ and $0 \mu$ g, respectively. The DNase I digests were run with the same probes that were chemically cleaved $(G+A$ lanes). The imperfect inverted repeat sequence to which AtrA-g binds is also shown.

results led us to speculate that binding of AtrA-g to the DNA fragment facilitated the binding of AdpA or that AtrA recruited AdpA to its binding sites. To test this speculation, we performed similar gel mobility shift assay using the DNA fragment containing the mS2 mutation in the AtrAg-binding sequence as the probe (Fig. $3 \mathrm{c}$ ). Because of the
mS2 mutation in the inverted repeat, no retarded signal was detected when an excess of AtrA-g was incubated with the probe (Fig. 3c, lane 6). Nevertheless, the binding of AdpA to probe $\mathrm{mS} 2$ was apparently enhanced in the presence of AtrA-g; when $0.1 \mu \mathrm{g}$ AdpA was used, no retarded signal was detected in the absence of AtrA-g 
(a)

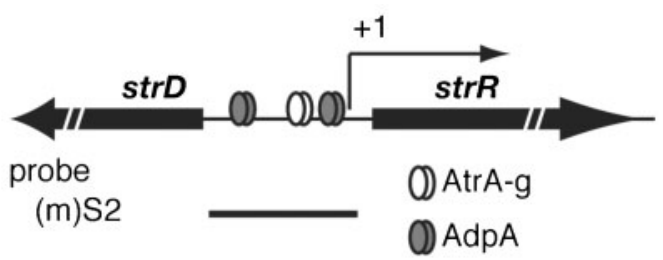

(b)

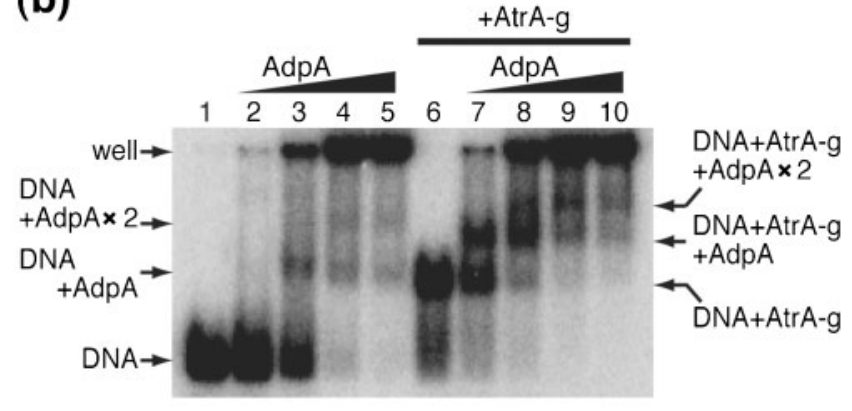

(c)

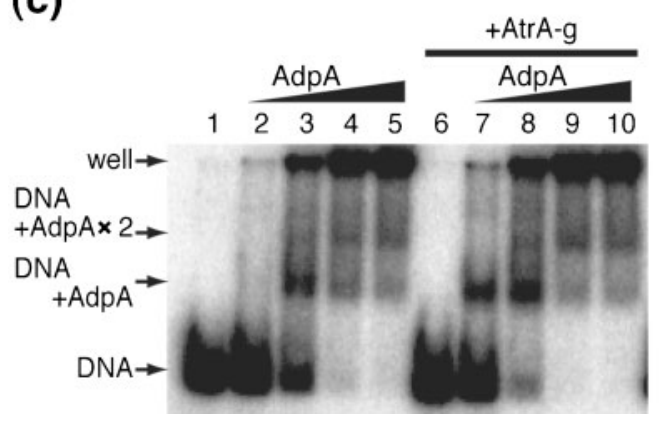

Fig. 3. Binding of AtrA-g and AdpA to the region upstream from the strR promoter. (a) Schematic diagrams of the strR promoter. The binding sites of AtrA-g and AdpA are shown. (b) Gel mobility shift assays with AtrA-g and AdpA. The ${ }^{32} \mathrm{P}$-labelled probe S2 (positions -344 to +19 ) containing the intact AtrA-g-binding sequence was used. The amounts of AdpA used in lanes 1, 2, 3, 4 and 5 were $0,0.1,0.2,0.4$ and $0.6 \mu \mathrm{g}$, respectively. In lanes 6,7 , 8, 9 and 10, $0.2 \mu \mathrm{g} \mathrm{AtrA-g}$ was used, in addition to the same amounts of AdpA as in lanes 1, 2, 3, 4 and 5, respectively. (c) Similar gel mobility shift assays using the mutated probe $\mathrm{mS} 2$ containing the mutations in the AtrA-g-binding sequence. The amounts of proteins were the same as those in (b).

(Fig. 3c, lane 2), whereas a distinct retarded signal by binding of AdpA was detected in the presence of AtrA-g (Fig. 3c, lane 7). Similar enhancement of AdpA binding was also observed with GriR, the pathway-specific transcriptional activator for the grixazone biosynthesis gene cluster (Higashi et al., 2007), indicating that the enhancement of AdpA binding was not specific to AtrA-g (data not shown). These observations made unlikely the possibility that binding of AtrA-g to probe S2 facilitates the in vitro binding of AdpA or recruits AdpA to its binding sites, but showed that the presence of even AtrA-g unbound to its binding site facilitated the AdpA binding in an unknown manner. One possible explanation is that
AtrA-g, probably together with BSA in the reaction buffer, increased the stability of AdpA in vitro.

\section{Streptomycin production by an atrA-g-disrupted strain}

To determine the possible role of atrA-g in streptomycin biosynthesis in vivo, atrA-g was inactivated in such a way that most of atrA-g was replaced with aphII. The correct disruption of atrA-g was checked by Southern hybridization with appropriate probes (data not shown). The atrA$g$-disrupted strain (mutant $\Delta a t r A-g$ ) grew normally and formed aerial mycelium and spores with the same timecourse as the wild-type strain on YMPD agar (data not shown). Mutant $\triangle a t r A-g$ produced a yellow pigment, grixazone, which is one of the secondary metabolites under the control of A-factor (Ohnishi et al., 2004), to the same extent as the wild-type strain (data not shown). We examined the ability to produce streptomycin of mutant $\triangle a t r A-g$ on Bennett agar without glucose. This medium has been routinely used for streptomycin production assay in our laboratory because, due to glucose catabolite repression, S. griseus produces streptomycin in a very small amount on Bennett agar containing $1 \%$ glucose. Mutant $\Delta a t r A-g$ produced streptomycin to the same extent as the wild-type strain (data not shown), indicating that AtrA-g was non-essential for streptomycin production and exerted no detectable effect on streptomycin production when examined with the strain grown on the routine agar medium. This is a vivid contrast to the fact that mutant $\triangle a d p A$ never produces streptomycin on any media.

We next examined streptomycin production by mutant $\triangle a t r A-g$ under some different culture conditions, because there was the possibility that AtrA-g might contribute to the transcription of strR, to some extent, under some specific culture conditions. First, we lowered the culture temperature. The wild-type strain and mutant $\triangle a t r A-g$ were cultivated for $1-7$ days at $18{ }^{\circ} \mathrm{C}$ on Bennett agar and streptomycin production was examined by a bioassay using $B$. subtilis as an indicator. No difference was observed between the amounts of streptomycin produced by the two strains (data not shown). Second, the osmotic pressure of the medium was increased by addition of $0.03-0.5 \mathrm{M}$ sucrose. As the sucrose concentration of the medium increased, the yields of streptomycin production reduced. However, there was no difference between the amounts of streptomycin produced by the two strains in any cases (data not shown). Finally, some different carbon sources (glucose, maltose and glycerol) were added to Bennett agar. On Bennett agar containing $1 \%$ glucose, no difference in streptomycin production was detected, although the yields were greatly reduced due to glucose catabolite repression. However, on Bennett agar containing either $1 \%$ maltose or glycerol, mutant $\triangle a t r A-g$ produced a smaller amount of streptomycin than the wild-type strain. Fig. 4 shows a typical result of the bioassay for streptomycin production of the two strains on Bennett agar containing $1 \%$ maltose. 


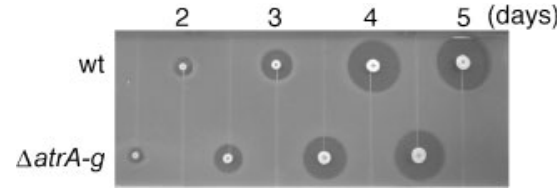

Fig. 4. Streptomycin production by the atrA-g-disrupted strain. The wild-type (wt) strain and mutant $\triangle$ atr $A-g$ were grown at $28^{\circ} \mathrm{C}$ on Bennett agar containing $1 \%$ maltose for the number of days indicated, and soft agar containing spores of $B$. subtilis was overlaid. The plates were incubated at $28{ }^{\circ} \mathrm{C}$ overnight, and streptomycin production was detected as a growth inhibition zone of the indicator around the colony.

The growth inhibition zones around the 4 and 5 day colonies of mutant $\triangle a t r A-g$ were somewhat smaller than those of the wild-type strain. We isolated four $\triangle a t r A-g$ strains independently and all of these strains showed a similar phenotype. We repeated this assay four times and calculated the amounts of streptomycin produced. The wild-type strain produced $3.7 \pm 1$ and $110 \pm 60 \mu \mathrm{g}$ streptomycin per colony on days 4 and 5 , respectively, whereas mutant $\triangle a t r A-g$ produced $0.89 \pm 0$ and $39 \pm 10 \mu \mathrm{g}$ streptomycin per colony on days 4 and 5 , respectively. The reduced streptomycin production by mutant $\triangle a t r A-g$ was partially complemented by pKU209-atrA-g carrying atrA-g with its own promoter on low-copy-number plasmid pKU209. Mutant $\triangle a t r A-g$ harbouring the empty vector pKU209 produced $0.54 \pm 1$ and $26 \pm 20 \mu \mathrm{g}$ streptomycin per colony on days 4 and 5, respectively (data not shown). On the other hand, mutant $\triangle a t r A-g$ harbouring pKU209atr $A-g$ produced $1.7 \pm 1$ and $52 \pm 30 \mu \mathrm{g}$ streptomycin per colony on days 4 and 5 , respectively (data not shown). The reduced streptomycin production of mutant $\triangle a t r A-g$ in comparison with the wild-type strain was also observed clearly when the strains were cultured on SMM minimum medium (data not shown). These data showed that AtrA-g exerted a positive effect on streptomycin production at least under some culture conditions.

Because the yield of streptomycin significantly reflects the strR transcriptional level (Tomono et al., 2005) and because AtrA-g binds within the upstream regulatory region of the strR promoter, AtrA-g affects the transcriptional level of $s t r R$, but not the diverging $\operatorname{str} D$. On the assumption that $s t r R$ transcription in mutant $\triangle a t r A-g$ might be reduced when grown on Bennett agar containing $1 \%$ maltose or glycerol, we determined transcription of strR in mutant $\triangle a t r A-g$ by S1 mapping. S1 mapping with RNA prepared from mutant $\triangle a t r A-g$ cells grown on Bennett agar without glucose showed that the extent of the $s t r R$ transcription was the same as in the wild-type strain (data not shown). This was in agreement with the finding that the yields of streptomycin produced by mutant $\Delta a t r A-g$ and the wild-type strain were the same on the routine agar medium. We then prepared RNA from mutant $\Delta$ atrA-g grown on Bennett agar with $1 \%$ maltose and performed similar S1 mapping. Contrary to our expectation, however, no detectable difference in the transcription of strR was observed between mutant $\Delta a t r A-g$ and the wild-type strain (data not shown). We assume that the difference in strR transcription between mutant $\triangle a \operatorname{tr} A-g$ and the wild-type strain was too small to be detected by the $S 1$ mapping method employed.

\section{DISCUSSION}

We thought that the four proteins which we previously found to bind the region upstream of the promoter of $s t r R$, the pathway-specific activator gene for the streptomycin biosynthesis gene cluster in S. griseus, would provide useful information on the regulation of streptomycin biosynthesis by A-factor. Because strR is the pathway-specific activator, a variety of external signals, such as nutrient conditions and physiological conditions, are assumed to be gathered to the strR promoter and to affect its transcription. One of the four proteins was AdpA, a key transcriptional activator in the A-factor regulatory cascade, which we have extensively and intensively studied. The recent discovery by Uguru et al. (2005) that AtrA-c of S. coelicolor A3(2) binds not only the flanking regions of the actII-ORF4 promoter but also the region upstream from the $s t r R$ promoter in a heterologous host reminded us of the proteins and led us to search for a homologue of AtrA-c in $S$. griseus and to study its possible role in the regulation of streptomycin biosynthesis. As expected, AtrA-g, which turns out to be the orthologue of AtrA-c in S. griseus, was identified and shown to bind the inverted repeat (positions -123 to -137 with respect to the transcriptional start point of $s t r R$ ) in front of the $s t r R$ promoter. We assume that AtrA-g corresponds to one of the four proteins we previously detected (Vujaklija et al., 1993), on the basis of the location of its binding site and its constitutive production independently of A-factor. Our careful experiments for determination of the role of AtrA-g showed that it affected streptomycin biosynthesis in a positive way under some specific conditions just as a tuner. We observed the positive effect of AtrA-g on streptomycin production only when the strains were grown on certain media, such as Bennett agar containing either $1 \%$ maltose or glycerol and SMM agar. The reason why the positive effect of AtrA-g on streptomycin production was observed only on some media is not clear. However, maltose or glycerol seem to have no direct relation with the function of AtrA-g, because mutant $\triangle a t r A-g$ produced a smaller amount of streptomycin than the wild-type strain even on SMM.

Hong et al. (2007) observed a negative effect of the $S$. coelicolor A3(2) atrA-c on streptomycin production by a heterologous host, S. griseus, and discussed the possibility that expression of atrA-c in S. griseus may cause DNAbinding-dependent reduction in streptomycin production and in the strR mRNA level. Our results obtained with atrA-g in the original host are apparently in contradiction 
to their observations: AtrA-g has a positive effect on streptomycin production, although the effect is only conditional and rather small. The reason why atrA-c exerts the negative effect on streptomycin production in the heterologous host $S$. griseus is unclear, but it may fail to exert the positive function that is observed for actinorhodin production in the original S. coelicolor A3(2) host. The negative effect of $a t r A-c$ on streptomycin production in $S$. griseus that Hong et al. (2007) observed may be due to interference of strR transcription resulting from competitive binding to the inverted repeat in front of the $s t r R$ promoter between the cognate AtrA-g and the heterologous, overproduced AtrA-c. Alternatively, different culture conditions or different $S$. griseus strains used by the two groups may explain this apparent contradiction. In any case, it is important and to be emphasized that the effect of AtrA-g on streptomycin production that we observed is much smaller than that discussed by Hong et al. (2007); the A-factor-ArpA-AdpA-StrR regulatory cascade is the major and decisive regulation for streptomycin production in $S$. griseus and atrA-g is a tuner under some specific conditions.

How does AtrA-g contribute to streptomycin production under these specific conditions? It is probable that coexistence of AdpA and AtrA-g enhances the transcriptional activation of strR by an as yet unclear mechanism. However, the level of the transcriptional enhancement by AtrA-g seems to be too low to be detected by S1 nuclease mapping, even with RNAs prepared from cells grown under the specific conditions. For full activation of the $\operatorname{str} R$ transcription, AdpA is required to occupy the two sites upstream from the strR promoter, at approximately nucleotide positions -270 and -50 (Tomono et al., 2005). AdpA recruits RNA polymerase to the promoter region of $s t r R$, and perhaps facilitates the isomerization of the RNA polymerase-DNA complex into an open complex competent for transcriptional initiation. In this process, we speculate that the DNA sequence upstream from the strR promoter bends so that the two molecules of AdpA dimer bound at positions -270 and -50 become accessible to the promoter region to recruit RNA polymerase. In fact, we showed that the DNA fragment upstream from the strR promoter contains multiple bent sites even in the absence of any DNA-binding proteins (Vujaklija et al., 1993). Because an AdpA dimer does bind the two AdpA-binding sites simultaneously with AtrA-g in such a way that ArtA-g sits between the two AdpA dimers, we speculate that AtrAg causes further DNA bending so that the AdpA dimer bound to the site at position -270 can interact with the AdpA dimer bound to the site at position -50. For full transcriptional activation of strR, the two AdpA-binding sites should be occupied by AdpA (Tomono et al., 2005), which suggests that the two AdpA dimers should interact with each other to assist RNA polymerase in initiating the transcription of strR. Uguru et al. (2005) also speculated a DNA bending caused by AtrA-c for transcriptional activation of actII-ORF4 (see below).
AtrA-c binds two sites in the flanking region of the actIIORF4 promoter at nucleotide positions -162 and +86 , both of which are not usual as the binding sites of transcriptional activators (Uguru et al., 2005). Concerning the transcriptional activation of actII-ORF4 by AtrA-c, Uguru et al. (2005) speculated that the DNA-bound AtrA-c might induce DNA bending in such a way that another transcriptional factor bound in the vicinity of the AtrA-cbinding site can readily make an interaction with RNA polymerase. AtrA-g bound the region upstream from the $s t r R$ promoter at approximately position -130 , which is also unusual for a transcriptional activator. Considering (i) the AtrA-g-binding site relative to the two AdpA-binding sites, (ii) simultaneous binding of AdpA and AtrA-g to the respective binding sites, and (iii) a conditionally positive effect of AtrA-g on streptomycin production, we assume that AtrA-g helps AdpA to initiate transcription of $s t r R$ in an unknown way. A speculative mechanism by which AtrA-g helps AdpA in transcriptional activation of $s t r R$ is DNA bending, as described above. Further analyses of AtrA-c in activation of the actII-ORF4 transcription and of AtrA-g in activation of the strR transcription will give us new insight into the function of the AtrA regulators for secondary metabolism.

\section{ACKNOWLEDGEMENTS}

S. Hirano was supported by the Japan Society for the Promotion of Science. This work was supported by a Grant-in-Aid for Scientific Research on Priority Areas 'Applied Genomics' from Monkasho.

\section{REFERENCES}

Ando, N., Ueda, K. \& Horinouchi, S. (1997). A Streptomyces griseus gene (sgaA) suppresses the growth disturbance caused by high osmolality and a high concentration of A-factor during early growth. Microbiology 143, 2715-2723.

Bentley, S. D., Chater, K. F., Cerdeño-Tárraga, A. M., Challis, G. L., Thomson, N. R., James, K. D., Harris, D. E., Quail, M. A., Kieser, H. \& other authors (2002). Complete genome sequence of the model actinomycete Streptomyces coelicolor A3(2). Nature 417, 141-147.

Fernández-Moreno, M. A., Caballero, J. L., Hopwood, D. A. \& Malpartida, F. (1991). The act cluster contains regulatory and antibiotic export genes, direct targets for translational control by the bldA tRNA gene of Streptomyces. Cell 66, 769-780.

Higashi, T., Iwasaki, Y., Ohnishi, Y. \& Horinouchi, S. (2007). A-factor and phosphate depletion signals are transmitted to the grixazone biosynthesis genes via the pathway-specific transcriptional activator GriR. J Bacteriol 189, 3515-3524.

Hirano, S., Kato, J., Ohnishi, Y. \& Horinouchi, S. (2006). Control of the Streptomyces subtilisin inhibitor gene by AdpA in the A-factor regulatory cascade in Streptomyces griseus. J Bacteriol 188, 6207-6216.

Hong, B., Phornphisutthimas, S., Tilley, E., Baumberg, S. \& McDowall, K. J. (2007). Streptomycin production by Streptomyces griseus can be modulated by a mechanism not associated with change in the adpA component of the A-factor cascade. Biotechnol Lett 29, 57-64.

Hopwood, D. A., Bibb, M. J., Chater, K. F., Kieser, T., Bruton, C. J., Kieser, H. M., Lydiate, D. J., Smith, C. P., Ward, J. M. \& Schrempf, H. 
(1985). Genetic Manipulation of Streptomyces: a Laboratory Manual. Norwich: The John Innes Foundation.

Horinouchi, S. (2002). A microbial hormone, A-factor, as a master switch for morphological differentiation and secondary metabolism in Streptomyces griseus. Front Biosci 7, d2045-d2057.

Horinouchi, S. (2007). Mining and polishing of the treasure trove in the bacterial genus Streptomyces. Biosci Biotechnol Biochem 71, 283-299.

Horinouchi, S. \& Beppu, T. (2007). Hormonal control by A-factor of morphological development and secondary metabolism in Streptomyces. Proc Jpn Acad Ser B 83, 277-295.

Horinouchi, S., Kumada, Y. \& Beppu, T. (1984). Unstable genetic determinant of A-factor biosynthesis in streptomycin-producing organisms: cloning and characterization. J Bacteriol 158, 481-487.

Kato, J., Chi, W.-J., Ohnishi, Y., Hong, S.-K. \& Horinouchi, S. (2005). Transcriptional control by A-factor of two trypsin genes in Streptomyces griseus. J Bacteriol 187, 286-295.

Kato, J., Funa, N., Watanabe, H., Ohnishi, Y. \& Horinouchi, S. (2007). Biosynthesis of $\gamma$-butyrolactone autoregulators that switch on secondary metabolism and morphological development in Streptomyces. Proc Natl Acad Sci U S A 104, 2378-2383.

Oh, S. H. \& Chater, K. F. (1997). Denaturation of circular or linear DNA facilitates targeted integrative transformation of Streptomyces coelicolor A3(2): possible relevance to other organisms. J Bacteriol 179, 122-127.

Ohnishi, Y., Kameyama, S., Onaka, H. \& Horinouchi, S. (1999). The A-factor regulatory cascade leading to streptomycin biosynthesis in Streptomyces griseus: identification of a target gene of the A-factor receptor. Mol Microbiol 34, 102-111.

Ohnishi, Y., Furusho, Y., Higashi, T., Chun, H.-K., Furihata, K., Sakuda, S. \& Horinouchi, S. (2004). Structures of grixazone A and B, A-factor-dependent yellow pigments produced under phosphate depletion by Streptomyces griseus. J Antibiot 57, 218-223.
Ohnishi, Y., Yamazaki, H., Kato, J., Tomono, A. \& Horinouchi, S. (2005). AdpA, a central transcriptional regulator in the A-factor regulatory cascade that leads to morphological development and secondary metabolism in Streptomyces griseus. Biosci Biotechnol Biochem 69, 431-439.

Retzlaff, L. \& Distler, J. (1995). The regulator of streptomycin gene expression, StrR, of Streptomyces griseus is a DNA binding activator protein with multiple recognition sites. Mol Microbiol 18, 151-162.

Strohl, W. R. (1992). Compilation and analysis of DNA sequences associated with apparent streptomycete promoters. Nucleic Acids Res 20, 961-974.

Tomono, A., Tsai, Y., Yamazaki, H., Ohnishi, Y. \& Horinouchi, S. (2005). Transcriptional control by A-factor of $s t r R$, the pathwayspecific transcriptional activator for streptomycin biosynthesis in Streptomyces griseus. J Bacteriol 187, 5595-5604.

Uguru, G. C., Stephens, K. E., Stead, J. A., Towle, J. E., Baumberg, S. \& McDowall, K. J. (2005). Transcriptional activation of the pathwayspecific regulator of the actinorhodin biosynthetic genes in Streptomyces coelicolor. Mol Microbiol 58, 131-150.

Vujaklija, D., Ueda, K., Hong, S.-K., Beppu, T. \& Horinouchi, S. (1991). Identification of an A-factor-dependent promoter in the streptomycin biosynthetic gene cluster of Streptomyces griseus. Mol Gen Genet 229, 119-128.

Vujaklija, D., Horinouchi, S. \& Beppu, T. (1993). Detection of an Afactor-responsive protein that binds to the upstream activation sequence of $s t r R$, a regulatory gene for streptomycin biosynthesis in Streptomyces griseus. J Bacteriol 175, 2652-2661.

Yamazaki, H., Ohnishi, Y. \& Horinouchi, S. (2000). An A-factordependent extracytoplasmic function sigma factor $\left(\sigma^{\mathrm{AdsA}}\right)$ that is essential for morphological development in Streptomyces griseus. J Bacteriol 182, 4596-4605.

Edited by: J.-H. Roe 